\title{
Neoclassical theory of rotation and electric field in high collisionality plasmas with steep gradients
}

\author{
H. A. Claassen, H. Gerhauser, and A. Rogister ${ }^{a}$ \\ Institut für Plasmaphysik, Forschungszentrum Jülich GmbH, Association EURATOM-FZJ, \\ Trilateral Euregio Cluster, D-52425, Jülich, Germany \\ C. Yarim \\ Istanbul Technical University, Faculty of Aerospace Sciences, 80626 Maslak/Istanbul, Turkey
}

(Received 22 February 2000; accepted 7 June 2000)

\begin{abstract}
The equation describing the radial transport of toroidal momentum in a collisional subsonic plasma with steep gradients has been obtained via a systematic expansion of the two-fluid equations. The diffusion rate is classical; the poloidal rotation, driven by the temperature gradient, generates, in turn, a toroidal flow gradient, also in Ohmic discharges. Moreover, important modifications of the parallel momentum equation are found to arise if $\Lambda_{1} \equiv\left(\nu_{i} / \Omega_{i}\right)\left(q^{2} R^{2} / r L_{T}\right)$ is $\geqslant O(1 / 3)$; the poloidal rotation velocity is then no longer unique but obeys a cubic equation which may allow for bifurcated equilibria under certain conditions. The toroidal velocities predicted for Ohmic discharges compare well with those measured in PLT [Princeton Large Torus; S. Suckewer et al., Nucl. Fusion 21, 1301 (1981)]; the relevance of the extended equation providing the poloidal rotation velocity to selected experimental edge plasmas is discussed. (C) 2000 American Institute of Physics. [S1070-664X(00)04009-X]
\end{abstract}

\section{INTRODUCTION}

The neoclassical theory of plasma poloidal and toroidal rotation has been a long evolving subject. Attention will be focused here on the high collisionality regime. The predictions of the various models that are described in the literature have to be relativized in view of (i) the scaling adopted with regard to the ratio of the toroidal flow velocity to the sound and ion thermal speeds $\left[c_{s} \sim c_{i}, c_{i} \equiv\left(T_{i} / m_{i}\right)^{1 / 2}\right]$; (ii) the formulation of the viscosity stress tensors: it is known indeed that Braginskii's expressions ${ }^{1}$ lead to $U_{\theta, i}=0$ for the poloidal velocity; this contrasts with the result $U_{\theta, i}$ $=k \partial_{r} T_{i} / e_{i} B_{\varphi} \quad$ obtained from a Vlasov-Boltzmann approach; ${ }^{2}$ Braginskii's stress tensors have therefore been corrected by Mikhailowskii and Tsypin; ${ }^{3}$ (iii) the selfconsistency of the assumptions that are introduced.

Hogan, ${ }^{4}$ Hinton and Wong, ${ }^{5}$ and Connor et al. ${ }^{6}$ studied the relaxation of toroidal flows with velocities of the order of the sound speed, starting from either Braginskii's two-fluid equation $^{4,6}$ or the Vlasov-Boltzmann equation. ${ }^{5}$ Hogan obtained an effective neoclassical diffusion coefficient,

$$
\mu_{\perp, i}=\eta_{2, i}\left(1+2.31 q^{2}\right)
$$

for the toroidal angular momentum, $\eta_{2, i}=1.2 P_{i} \nu_{i} / \Omega_{i}^{2}$ being the second Braginskii's perpendicular viscosity coefficient, and $q$ the safety factor (the definition of the collision frequency $\nu_{i}$ is that of Ref. 1 and $\left.\Omega_{i}=e_{i} B / m_{i}\right)$. By contrast, Hinton and Wong and Connor et al. obtained the classical rate,

$$
\mu_{\perp, i}=\eta_{2, i}
$$

${ }^{\text {a)} E l e c t r o n i c ~ m a i l: ~ a . r o g i s t e r @ f z-j u e l i c h . d e ~}$
The difference probably arises because Hogan imposed (artificially) the constraint of pure toroidal flows. The agreement between the two other groups of authors is a consequence of that the Mikhailowskii-Tsypin corrections to Braginskii's stress tensors are negligible if $U_{\varphi, i}$ $\gg T_{i} \partial_{r} \ln N_{i} / e_{i} B_{\theta}$ and $\partial_{r} P_{i} / N_{i} e_{i} B_{\theta}$.

Radial transport of toroidal momentum in tokamaks ${ }^{7-9}$ often occurs at rates that are faster than the classical rate by two orders of magnitude. In view of this, Stacey and Sigmar ${ }^{10}$ undertook showing that the gyrostresses, as modified by toroidal geometry effects, are of the proper magnitude to explain the momentum confinement time inferred from experiments. Subsequently, Stacey ${ }^{11}$ and Rogister ${ }^{12}$ allowed for short gradient lengthscales, i.e., $L_{\perp} \ll q R$, in accordance with the conditions prevailing at the plasma edge and in the vicinity of transport barriers. Those theories, however, failed to consider the Mikhailowskii-Tsypin corrections ${ }^{3}$ to the Braginskii stress tensor and thereby largely overestimated the role of gyroviscosity, as will be explained in the next sections. More recently, Claassen and Gerhauser obtained the classical toroidal momentum transport equation, ${ }^{13}$

$$
\begin{aligned}
m_{i} N_{i} \frac{\partial U_{\varphi, i}}{\partial t}= & \frac{\partial}{\partial r}\left[\eta_{2, i}\left(\frac{\partial U_{\varphi, i}}{\partial r}-\frac{0.107 q^{2}}{1+Q^{2} / S^{2}} \frac{\partial \ln T_{i}}{\partial r} \frac{B_{\varphi}}{B_{\theta}} U_{\theta, i}\right)\right] \\
& +m_{i} N_{i}\left(\dot{m}_{\varphi, i}-\nu_{\mathrm{cx}} U_{\varphi, i}\right)+J_{r} B_{\theta}
\end{aligned}
$$

for tokamak plasmas with circular cross sections and $L_{\perp} \ll r$ $\ll q R . \dot{m}_{\varphi, i}$ represents the average toroidal ion acceleration by an external momentum source (associated with, e.g., neutral beams); $-\nu_{\mathrm{cx}} U_{\varphi, i}$ describes the slowing down of the rotation via charge exchange with cold recycling neutrals; $J_{r} B_{\theta}$ is the torque exerted on the plasma in experiments with a polarization current. ${ }^{14,15}$ The quantities $Q$ and $S$ have been defined in Ref. 12: 


$$
\begin{aligned}
& Q=\left[4 B_{\varphi} U_{\theta, i}-2.5\left(T_{i} / e_{i}\right) \partial \ln N_{i}^{2} T_{i} / \partial r\right] B^{-1}, \\
& S=\left(2 r \chi_{\|, i} N_{i}^{-1}\right) / q^{2} R^{2}
\end{aligned}
$$

$\left(\chi_{\|, i}=3.9 P_{i} / m_{i} \nu_{i}\right.$ is the parallel heat diffusion coefficient $\left.{ }^{1}\right)$. The first term on the right-hand side of Eq. (1) arises from the perpendicular stress tensor; it has been obtained previously in Refs. 5 and 6 . The second term arises from the gyrostress tensor and is novel; it acts as a toroidal momentum source also in an Ohmic plasma; the exact condition under which it can be neglected in the initial relaxation of a given toroidal velocity profile can be explicited from Eq. (1). Worth noting is that the ratio of the first two terms is independent of the collision frequency, although the ratio $\eta_{2, i} / \eta_{3, i}$ of the perpendicular and gyroviscosity coefficients ${ }^{1}$ is $2.4 \nu_{i} / \Omega_{i}$. This apparent paradox is resolved by noting that the density, e.g., can be written in the form $^{12}$ $N_{i}=N_{i}^{(0)}(r)\left[1+n_{\cos }^{(1)} \cos \theta+n_{\sin }^{(1)} \sin \theta\right] \quad$ with $\quad n_{\cos }^{(1)} / n_{\text {sin }}^{(1)}$ $=-\left(B_{\varphi} / B\right) Q / S \propto \nu_{i} / \Omega_{i}$ and $n_{\cos }^{(1)}, n_{\text {sin }}^{(1)} \ll 1$.

The generalization of Eq. (1) to large aspect ratio tokamak plasmas with arbitrary cross sections will be given in the next sections. In order to complete the picture of plasma rotation, an equation for the poloidal velocity will also be derived. For tokamak plasmas with $L_{\perp} \ll r \ll q R$ and circular cross sections, it can be written as

$$
\begin{aligned}
U_{\theta, i}+ & 1.833\left(e_{i} B_{\varphi}\right)^{-1} \frac{\partial T_{i}}{\partial r} \\
= & 0.36 \frac{\eta_{2, i} / \eta_{0, i}}{1+Q^{2} / S^{2}} q^{2} R^{2} \frac{e_{i} B_{\varphi}}{T_{i}} \frac{\partial \ln T_{i}}{\partial r}\left[\frac{T_{i}}{e_{i} B_{\theta}} \frac{\partial U_{\varphi, i}}{\partial r}\right. \\
& +\frac{1}{2} U_{\varphi, i}^{2}-U_{\varphi, i} \frac{B_{\varphi}}{B_{\theta}}\left(U_{\theta, i}-\frac{T_{i}}{e_{i} B_{\varphi}} \frac{\partial \ln N_{i}^{2} T_{i}}{\partial r}\right) \\
& \left.+1.90 \frac{B_{\varphi}^{2}}{B_{\theta}^{2}}\left(U_{\theta, i}-0.8 \frac{T_{i}}{e_{i} B_{\varphi}} \frac{\partial \ln N_{i}^{1.6} T_{i}}{\partial r}\right)^{2}\right] \\
& -\frac{2 R^{2}}{3 \eta_{0, i}} J_{r} B_{\varphi}
\end{aligned}
$$

$\left(\eta_{0, i}=0.96 P_{i} / \nu_{i}\right.$ is the parallel viscosity coefficient $\left.{ }^{1}\right)$. The standard neoclassical result $U_{\theta, i}=-1.833\left(e_{i} B_{\varphi}\right)^{-1} \partial T_{i} / \partial r$ is therefore invalid, if either $r\left|L_{T}\right| / 3 q^{2} R^{2} \leqslant \nu_{i} / \Omega_{i}$, where $L_{T}=\left(\partial \ln T_{i} / \partial r\right)^{-1}$ or $0.3 \hat{\nu}_{i} U_{\varphi, i} / c_{i}>1$, where $\hat{\nu}_{i}=q R \nu_{i} / c_{i}$; we note that the plasma is in the high collisionality regime if $^{16} \hat{\nu}_{i}>0.222$.

Equations (1) and (2) are amenable to a cubic equation for $U_{\theta, i}$ for prescribed temperature and density profiles. The poloidal flow velocity can therefore assume either one or three different values, depending on the experimental conditions. This conclusion is reminiscent of the multivalued behavior of the neoclassical heat flux with respect to $L_{T}$ obtained earlier in the framework of the revisited neoclassical theory ${ }^{17,18}$ when $q>5$. It suggests also the possibility of bifurcated rotation profiles.

As usual, the radial electric field finally obtains from the radial momentum balance equation,

$$
E_{r}=B_{\theta} U_{\varphi, i}-B_{\varphi} U_{\theta, i}+\left(T_{i} / e_{i}\right) \partial \ln P_{i} / \partial r .
$$

The remainder of this paper is organized as follows. The general formulation of the ambipolarity constraint and of a suitably weighted flux surface average of the parallel momentum equation are presented in Sec. II; the ordering scheme that we shall adopt is then discussed. The explicit ambipolarity constraint and parallel momentum equation, simplified self-consistently following the ordering scheme, are obtained in Secs. III and IV for arbitrary cross sections [Eqs. (16) and (22)]. After briefly summarizing our results, we compare in Sec. $\mathrm{V}$ the predictions of the theory with experimental data on toroidal rotation in PLT (Princeton Large Torus) ${ }^{7}$ Ohmic discharges; we show further the relevance of the novel terms obtained in the parallel momentum equation in predicting correctly the poloidal rotation velocity in high-density discharges. Since the Mikhailovskii-Tsypin modifications of the stress tensor play such an important role, an outline of the underlying theory is presented in the Appendix.

\section{GENERAL FORMULATION OF THE PROBLEM AND ORDERING}

We consider an electron-ion plasma with $e_{i}=-e_{e}=e$ and hence $N_{e}=N_{i}$. We shall be concerned with the projections of the momentum equations, summed over both species, onto the toroidal and the parallel (to $\mathbf{B}$ ) directions, viz.,

$$
\left[\boldsymbol{\nabla} \cdot \boldsymbol{\Pi}_{i}+m_{i} N_{i}\left(\partial_{t}+\mathbf{U}_{i} \cdot \boldsymbol{\nabla}\right) \mathbf{U}_{i}\right] \cdot \hat{e}_{\varphi}=B_{\chi} J_{\psi}
$$

and

$$
\left[\boldsymbol{\nabla}\left(P_{e}+P_{i}\right)+\boldsymbol{\nabla} \cdot \boldsymbol{\Pi}_{i}+m_{i} N_{i}\left(\partial_{t}+\mathbf{U}_{i} \cdot \boldsymbol{\nabla}\right) \mathbf{U}_{i}\right] \cdot \hat{n}=0 .
$$

$\boldsymbol{\Pi}_{i}$ is the ion stress tensor (see Appendices A and B); $P_{e}$ and $P_{i}$ are the electron and ion scalar pressures; $\hat{n} \equiv \mathbf{B} / B$ is the unit vector tangent to the local magnetic field line; $\psi, \chi$, and $\varphi$ are the toroidal flux surface coordinate, the generalized poloidal angle and the toroidal angle, respectively. We have considered axisymmetric plasmas $(\partial / \partial \varphi \equiv 0$ when applied to scalars) in the limit $m_{e} / m_{i} \rightarrow 0$; in that approximation we may consider that $\partial T_{e} / \partial \chi \equiv 0$. Dividing Eq. (6) by $B_{\chi}$ and integrating over a flux surface yields the ambipolarity constraint,

$$
\begin{aligned}
& \oint h_{\varphi} h_{\chi} B_{\chi}^{-1}\left[\boldsymbol{\nabla} \cdot \boldsymbol{\Pi}_{i}+m_{i} N_{i}\left(\partial_{t}+\mathbf{U}_{i} \cdot \boldsymbol{\nabla}\right) \mathbf{U}_{i}\right] \cdot \hat{e}_{\varphi} d \chi \\
& =\oint h_{\varphi} h_{\chi} J_{\psi} d \chi .
\end{aligned}
$$

The left-hand side of Eq. (8) vanishes if there is no current injection. A second one-dimensional equation is obtained by integrating the product of Eq. (7) with $B h_{\psi}$ over a flux surface:

$$
\oint J B\left[\boldsymbol{\nabla} \cdot \boldsymbol{\Pi}_{i}+m_{i} N_{i}\left(\partial_{t}+\mathbf{U}_{i} \cdot \boldsymbol{\nabla}\right) \mathbf{U}_{i}\right] \cdot \hat{n} d \chi=0 .
$$

Indeed, $\hat{n} \cdot \boldsymbol{\nabla} \equiv\left(B_{\chi} / B\right) h_{\chi}^{-1} \partial_{\chi}$; hence $\oint h_{\varphi} h_{\chi} B h_{\psi} \hat{n} \cdot \nabla P d \chi$ $=-\oint P \partial_{\chi}\left(h_{\varphi} h_{\psi} B_{\chi}\right) d \chi \equiv 0 \quad$ in view of $\boldsymbol{\nabla} \cdot \mathbf{B}=J^{-1}$ $\times \partial_{\chi}\left(h_{\varphi} h_{\psi} B_{\chi}\right)=0 . J$ is the Jacobian of the transformation $d \mathbf{r} \rightarrow h_{\psi} d \psi \hat{e}_{\psi}+h_{\chi} d \chi \hat{e}_{\chi}+h_{\varphi} d \varphi \hat{e}_{\varphi} ;$ in the following, $h_{\psi}$ $=1 / h_{\varphi} B_{\chi}$, where $h_{\varphi}=R$ is the major radius; $h_{\chi}$ is to be obtained from an equilibrium calculation. 
Equation (8) can be simplified by noting that $\boldsymbol{\nabla} \hat{e}_{\varphi}$ $=-\hat{e}_{\varphi} \nabla \ln h_{\varphi} ;$ hence

$$
\begin{aligned}
\left(\mathbf{U}_{i} \cdot \boldsymbol{\nabla} \mathbf{U}_{i}\right) \cdot \hat{e}_{\varphi} & =\mathbf{U}_{i} \cdot \boldsymbol{\nabla} U_{\varphi, i}-\left(\mathbf{U}_{i} \cdot \boldsymbol{\nabla} \hat{e}_{\varphi}\right) \cdot \mathbf{U}_{i} \\
& =h_{\varphi}^{-1}\left(h_{\chi}^{-1} U_{\chi, i} \partial_{\chi}+h_{\psi}^{-1} U_{\psi, i} \partial_{\psi}\right) h_{\varphi} U_{\varphi},
\end{aligned}
$$

from which it follows that the inertia term in Eq. (8) can be transformed into

$m_{i} \oint h_{\varphi} J \partial_{t}\left(N_{i} U_{\varphi, i}\right) d \chi+m_{i} \partial_{\psi} \oint h_{\varphi} J h_{\psi}^{-1} N_{i} U_{\varphi, i} U_{\psi, i} d \chi$.

We have made use of $\boldsymbol{\nabla} \cdot\left(N_{i} \mathbf{U}_{i}\right)=-\partial_{t} N_{i}$; the last term is the divergence of the radial flux of angular momentum. Noting further that the various stress tensors are of the form

$$
\Pi=\boldsymbol{\alpha} \boldsymbol{\beta}+\boldsymbol{\beta} \boldsymbol{\alpha}
$$

(see Appendix A), it is easily shown by an analogous procedure that

$$
\oint h_{\varphi} h_{\chi} B_{\chi}^{-1}\left(\boldsymbol{\nabla} \cdot \boldsymbol{\Pi}_{i}\right) \cdot \hat{e}_{\varphi} d \chi=\partial_{\psi} \oint h_{\varphi} J h_{\psi}^{-1} \Pi_{\psi \varphi} .
$$

The ambipolarity constraint (8) can therefore be rewritten as

$$
\begin{aligned}
\partial_{t} \oint & J h_{\varphi} m_{i} N_{i} U_{\varphi, i} d \chi+\partial_{\psi} \oint h_{\psi}^{-1} J h_{\varphi} \\
& \times\left(m_{i} N_{i} U_{\varphi, i} U_{\psi, i}+\Pi_{\varphi \psi}\right) d \chi=\oint h_{\psi}^{-1} J J_{\psi} d \chi .
\end{aligned}
$$

With the ordering adopted both here and in Ref. 12 [cf. Eq. (12)], the contributions arising from the density evolution and from the radial flux of angular momentum are negligible in the framework of the neoclassical theory of a one ion species plasma.

Equation (9) can also be transformed by noting that, in view of (10):

$$
\begin{aligned}
\oint J B & \left(\nabla \cdot \Pi_{i}\right) \cdot \hat{n} d \chi \\
= & \partial_{\psi} \oint J B h_{\psi}^{-1} \Pi_{\psi \|} d \chi-\oint J\left(\Pi_{\psi \|} h_{\psi}^{-1} \partial_{\psi} B\right. \\
& \left.+\Pi_{\chi \|} h_{\chi}^{-1} \partial_{\chi} B\right) d \chi-\oint J B\left[\hat{p} \cdot \nabla \hat{n} \cdot \hat{p} \Pi_{\psi \psi}\right. \\
& +\hat{p} \cdot \nabla \hat{n} \cdot \hat{b} \Pi_{\psi \beta}+\hat{b} \cdot \nabla \hat{n} \cdot \hat{p} \Pi_{\beta \psi}+\hat{b} \cdot \nabla \hat{n} \cdot \hat{b} \Pi_{\beta \beta} \\
& \left.+\hat{n} \cdot \nabla \hat{n} \cdot \hat{p} \Pi_{\| \psi}+\hat{n} \cdot \nabla \hat{n} \cdot \hat{b} \Pi_{\| \beta}\right] .
\end{aligned}
$$

Here, $\hat{p} \equiv \hat{e}_{\psi}$ is the normal to the flux surface and $\hat{b} \equiv \hat{n} \times \hat{p}$ $=\left(B_{\varphi} / B\right) \hat{e}_{\chi}-\left(B_{\chi} / B\right) \hat{e}_{\varphi}$ is the binormal to the field line. The Christoffel symbols in the coordinate system $\hat{p}, \hat{b}, \hat{n}$ have been given in Appendix A of Ref. 19 [an error was made in transcribing Eq. (A8), which should read as $\left.\hat{b} \cdot \boldsymbol{\nabla} \hat{b} \cdot \hat{n}=\hat{n} \cdot \boldsymbol{\nabla} \ln \left(B h_{\psi}\right)\right]$. Worth noting is that $\hat{p} \cdot \boldsymbol{\nabla} \hat{n} \cdot \hat{p}$ $=-\hat{b} \cdot \boldsymbol{\nabla} \hat{n} \cdot \hat{b}-\hat{n} \cdot \boldsymbol{\nabla} \ln B \quad$ (and, of course, $\hat{b} \cdot \boldsymbol{\nabla} \hat{n} \cdot \hat{b}=-\hat{b}$ $\cdot \boldsymbol{\nabla} \hat{b} \cdot \hat{n}), \quad \Pi_{\psi \beta}=\Pi_{\beta \psi}, \quad \hat{p} \cdot \boldsymbol{\nabla} \hat{n} \cdot \hat{b}+\hat{b} \cdot \boldsymbol{\nabla} \hat{n} \cdot \hat{p}=-\left(B_{\varphi} B_{\chi} / B^{2}\right) \hat{p}$ $\cdot \nabla \ln \nu$, where $\nu \equiv h_{\chi} B_{\varphi} / h_{\varphi} B_{\chi}$ is the local pitch angle of the magnetic field line, $\hat{n} \cdot \boldsymbol{\nabla} \hat{n} \cdot \hat{b}=\hat{b} \cdot \boldsymbol{\nabla} \ln B$, and $\hat{n} \cdot \nabla \hat{n} \cdot \hat{p}$ $=\hat{p} \cdot \nabla \ln B+\mu_{0} B^{-2} \hat{p} \cdot \nabla\left(P_{e}+P_{i}\right) \quad$ the low $\beta \equiv 2 \mu_{0}\left(P_{e}\right.$ $\left.+P_{i}\right) / B^{2}$ approximation will be considered here and the last term neglected]; last, $\alpha_{\beta}$ stands for $\boldsymbol{\alpha} \cdot \hat{b}$. The contribution of the inertia term to Eq. (9) can finally be cast into the form

$$
\begin{aligned}
\oint J B & {\left[m_{i} N_{i}\left(\partial_{t}+\mathbf{U}_{i} \cdot \boldsymbol{\nabla}\right) \mathbf{U}_{i}\right] \cdot \hat{n} d \chi } \\
= & \oint J B m_{i} N_{i} \partial_{t} U_{\|, i} d \chi+\oint m_{i} N_{i}\left(B / B_{\chi}\right)\left[U_{\chi, i} \partial_{\chi} U_{\|, i}\right. \\
& \left.+U_{\beta, i}^{2}\left(B_{\chi} / B\right) \partial_{\chi} \ln B h_{\psi}-U_{\|, i} U_{\beta, i}\left(B_{\varphi} / B\right) \partial_{\chi} \ln B\right] .
\end{aligned}
$$

Information on the poloidal angle dependence of the profiles $N_{i}(\psi, \chi), T_{i}(\psi, \chi), U_{\|, i}(\psi, \chi)$, and $V(\psi, \chi)$ ( $V$ is the electrostatic potential) is required to carry out the integrals on the left-hand side of Eq. $\left(8^{\prime}\right)$ and on the right-hand sides of Eqs. $\left(9^{\prime}\right)$ and $\left(9^{\prime \prime}\right)$. This has been obtained in Ref. 12 by expanding the basic two-fluid equations according to the ordering

$$
\frac{r}{q R} \sim \frac{L_{\perp}}{r} \sim\left(\frac{a_{i}}{L_{\perp}}\right)^{1 / 2} \sim \frac{1}{\hat{\nu}_{i}} \sim \mu \ll 1
$$

[typically, $\mu \sim 0.1$; the assumption $\hat{\nu}_{i} \equiv q R \nu_{i} / c_{i} \gg 1$ is to validate the use of the two-fluid equations (high collisionality regime)]. It was found that the scaling,

$$
U_{\|, i} \sim U_{\varphi, i} \sim \mu c_{i}, \quad U_{\beta, i} \sim U_{\chi, i} \sim \mu^{2} c_{i}, \quad U_{\psi, i} \sim \mu^{6} c_{i},
$$

follows automatically from (11); another consequence of (11) is that the profiles of density, temperature, pressure, electrostatic potential, and of the various velocity components can be expanded as

$$
F(\psi, \chi)=F^{(0)}(\psi)+\mu F^{(1)}(\psi, \chi)+\cdots,
$$

with $\oint F^{(1),(2), \ldots}(\psi, \chi) d \chi=0$. The relations between $N_{i}^{(1)}$, $T_{i}^{(1)}, P_{i}^{(1)}, V^{(1)}, U_{\|, i}^{(2)}$, and $B^{(1)}=B-B^{(0)}(\psi)$ are given for arbitrary cross sections in Eqs. (45)-(48) and (52) of Ref. 12; explicit results for circular cross sections are shown in Eqs. (63) and (64). [Note: we have written $U_{\|, i}=\mu U_{\|, i}^{(1)}(\psi)$ $+\mu^{2} U_{\|, i}^{(2)}(\psi, \chi)+\cdots$ as $U_{\|, i} \sim \mu c_{i}$; the equations of Ref. 12 quoted above are independent of the model adopted for the stress tensors.] The function $B(\psi, \chi)$ can be considered as known for our purposes. We finally stress that the scaling (11) implies that $r\left|L_{T}\right| / q^{2} R^{2} \sim \nu_{i} / \Omega_{i}$; leading-order modifications to the standard neoclassical result for $U_{\chi, i}$ will therefore be obtained [see the remark below Eq. (4)].

Toroidal velocities roughly of the order of the sound speed are observed in discharges with high-power neutral beam injection. The scaling $U_{\|, i} \sim U_{\varphi, i} \sim c_{i}$ would, however, lead to results identical to those of Hinton and Wong ${ }^{5}$ and of Connor et al. ${ }^{6}$-i.e., to an equation less general than Eq. (1) - unless we also upscale $a_{i} / L_{\perp}$ to order $\mu$ (in order that $\left.U_{\theta, i} \sim \mu c_{i}\right)$. Such a new ordering would presumably also increase the radial flux of angular momentum in Eq. (8) to values comparable with those of the other terms. Hence it is of interest for further work.

\section{EXPLICIT AMBIPOLARITY CONSTRAINT}

The parallel stress tensor does not contribute to the ambipolarity equation $\left(8^{\prime}\right)$ since $\left(\Pi_{0, i}\right)_{\varphi \psi}=0$, see Eq. (A1); this 
result has been attributed by Rutherford ${ }^{20}$ to the conservation of momentum in elastic collisions. The contributions from the components $\left(\Pi_{3-4, i}\right)_{\varphi \psi}$ and $\left(\Pi_{1-2, i}\right)_{\varphi \psi}$ can be easily evaluated in leading order with the help of Eqs. (A2) and (A3). Thus Eq. ( $\left.8^{\prime}\right)$ becomes

$$
\begin{aligned}
m_{i} \oint & J h_{\varphi} N_{i} \partial_{t} U_{\varphi, i} d \chi-\oint h_{\varphi} h_{\chi} J_{\psi} d \chi \\
= & \left(m_{i} / e_{i}\right) \partial_{\psi}\left(h_{\varphi}^{2} B_{\varphi}^{2} \oint B^{-4}\left[P_{i} \partial_{\chi}\left(U_{\|, i} B\right)+1.6 q_{\|, i} \partial_{\chi} B\right]\right. \\
& \times d \chi+1.2 h_{\varphi}^{2} B_{\varphi}^{2} \oint h_{\varphi} h_{\chi}\left(\nu_{i} / \Omega_{i}\right) \\
& \left.\times\left(B_{\chi} / B_{\varphi}\right) B^{-2} P_{i} \partial_{\psi} U_{\|, i} d \chi\right) .
\end{aligned}
$$

The first term on the right-hand side, respectively, the second term, is the contribution from the gyroviscous tensor, respectively, from the perpendicular stress tensor. The integrals can be simplified by making use of the expansion (13) and the results of Ref. 12. Hence

$$
\begin{aligned}
2 \pi m_{i} J h_{\varphi} N_{i} \frac{\partial U_{\varphi, i}}{\partial t}-\oint h_{\varphi} h_{\chi} J_{\psi} d \chi & \\
= & \frac{m_{i}}{e_{i}} h_{\varphi}^{2} B_{\varphi}^{2} \frac{\partial}{\partial \psi}\left\{2 \frac { P _ { i } } { B ^ { 3 } } \left[h _ { \varphi } B \frac { T _ { i } } { e _ { i } B } \left(3 e_{i} \frac{\partial V}{T_{i} \partial \psi}\right.\right.\right. \\
& \left.\left.+\frac{\partial \ln T_{i} N_{i}^{-1}}{\partial \psi}\right)+3 U_{\|, i}\right] \oint \frac{\partial n^{(1)}}{\partial \chi} b^{(1)} d \chi \\
& -12.48 \frac{P_{i}}{B^{3}} \frac{T_{i}}{m_{i} \nu_{i}} \frac{B_{\chi}}{B} h_{\chi}^{-1} \oint \frac{\partial^{2} n^{(1)}}{\partial \chi^{2}} b^{(1)} d \chi \\
& \left.+1.2 \frac{P_{i}}{B^{2}} \frac{\nu_{i}}{\Omega_{i}} \frac{h_{\chi}}{B_{\varphi}} h_{\psi}^{-1} \frac{\partial U_{\|, i}}{\partial \psi}\right\} .
\end{aligned}
$$

Here, $n^{(1)}=\mu N^{(1)}(\psi, \chi) / N^{(0)}(\psi)$ and $b^{(1)}=\mu B^{(1)}(\psi, \chi) /$ $B^{(0)}(\psi)$; all other quantities appearing in Eq. $\left(14^{\prime}\right)$ are leading-order ones, i.e., functions of $\psi$ only. The first and third terms on the right-hand side arise from Braginskii's gyro- and perpendicular stress tensors; the second termwhere we have replaced $\partial_{\chi} t_{i}^{(1)}$ by $-2 \partial_{\chi} n^{(1)}$ [cf. Eq. (45) in Ref. 12; this relation is a consequence of constant pressure along the field lines: $\partial\left(p_{e}^{(1)}+p_{i}^{(1)}\right) / \partial \chi=0$ and of electron adiabaticity: $\left.\quad \partial t_{e}^{(1)} / \partial \chi=0\right]$-is inherent to the Mikhailowskii-Tsypin correction of the gyrostresses. The relation between $\partial_{\chi}^{2} n^{(1)}$ and $\partial_{\chi} n^{(1)}$ follows directly from Eq. (52) of Ref. 12:

$$
\begin{aligned}
2 \chi_{\|, i} & \frac{B_{\chi}}{B} h_{\chi}^{-1} \frac{\partial^{2} n^{(1)}}{\partial \chi^{2}} \\
= & N_{i}\left[h_{\varphi} B_{\varphi} \frac{T_{i}}{e_{i} B}\left(4 e_{i} \frac{\partial V}{T_{i} \partial \psi}+\frac{\partial \ln T_{i}^{3 / 2} N_{i}^{-1}}{\partial \psi}\right)+4 U_{\|, i}\right] \\
& \times \frac{\partial n^{(1)}}{\partial \chi}+5 h_{\varphi} B_{\varphi} \frac{P_{i}}{e_{i} B} \frac{\partial \ln T_{i}}{\partial \psi} \frac{\partial b^{(1)}}{\partial \chi} .
\end{aligned}
$$

As a consequence, the first two terms on the right-hand side of Eq. $\left(14^{\prime}\right)$ have a similar structure; adding yields

$$
\begin{aligned}
2 \pi m_{i} J h_{\varphi} N_{i} \partial_{t} U_{\varphi, i}-\oint h_{\varphi} h_{\chi} J_{\psi} d \chi & \\
= & \left(m_{i} / e_{i}\right) h_{\varphi}^{2} \partial_{\psi}\left[-0.4 P_{i}\left(U_{\chi, i} / B_{\chi}\right) \oint b^{(1)} \partial_{\chi} n^{(1)} d \chi\right. \\
& \left.+1.2(2 \pi) P_{i}\left(\nu_{i} / \Omega_{i}\right) h_{\chi} B^{-1} h_{\psi}^{-1} \partial_{\psi} U_{\varphi, i}\right] .
\end{aligned}
$$

It is remarkable that $0.4 U_{\chi, i}$ results from the following combination of terms:

$$
\begin{aligned}
0.4 B_{\varphi} U_{\chi, i}= & (6.4-6)\left(h_{\psi}^{-1} \partial_{\psi} V+B_{\chi} U_{\varphi, i}\right)+\left(T_{i} / e_{i}\right) \\
& \times h_{\psi}^{-1}\left[(2.4-2) \partial_{\psi} \ln T_{i}+(-1.6+2) \partial_{\psi} \ln N_{i}\right],
\end{aligned}
$$

where the first, respectively the second, coefficients $[(6.4$, $2.4,-1.6)$, respectively $(-6,-2,+2)]$, arise from Braginskii's stress tensor, respectively the Mikhailovskii-Tsypin corrections. Braginskii's formulation therefore leads to an overestimate of the gyroviscous effects by a factor of order $6 / 0.4=15$ ! [We have noted that $U_{\varphi}=B_{\varphi} U_{\|} / B+O\left(\mu^{2}\right)$ and $\partial \ln B \sim \mu^{2} \partial_{\psi} \ln P$ within the framework of our ordering.] Appropriate forms of $b^{(1)}(=-\epsilon \cos \chi)$ and $n^{(1)}$ for circular cross-section tokamaks, in particular,

$$
n_{\text {sin }}^{(1)}=-5 \epsilon\left(1+Q^{2} / S^{2}\right)^{-1}\left(e_{i} B_{\varphi} S\right)^{-1} h_{\psi}^{-1} \partial_{\psi} T_{i}
$$

[see Eqs. (63) and (64) of Ref. 12], yields the ambipolarity equation (1) given in the introductory section; we have defined $n^{(1)}=n_{\cos }^{(1)} \cos \chi+n_{\text {sin }}^{(1)} \sin \chi$ and $\epsilon=r / R$.

\section{EXPLICIT PARALLEL MOMENTUM EQUATION}

The evaluation of the various contributions to Eq. (9), the (weighted) parallel momentum equation, is clearly a technical matter. Since, however, modifications to the conventional neoclassical result when

$$
\Lambda_{1} \equiv \frac{\nu_{i}}{\Omega_{i}} \frac{q^{2} R^{2}}{r L_{\perp}} \sim 1,
$$

are obtained here for the first time, we shall provide separately the different terms in the framework of our expansion scheme.

(i) The contribution from the parallel stress tensor is

$$
\begin{aligned}
\oint J B & \nabla \cdot \Pi_{0, i} \cdot \hat{n} d \chi \\
= & 3 \eta_{0, i} h_{\chi}^{-1}\left[U_{\chi, i}+1.83\left(e_{i} B_{\varphi}\right)^{-1} h_{\psi}^{-1} \partial_{\psi} T_{i}\right] \\
& \times \oint\left(\partial_{\chi} b^{(1)}\right)^{2} d \chi-0.123 m_{i} N_{i} \\
& \times\left[3.69 U_{\chi, i}-1.56\left(T_{i} / e_{i} B_{\varphi}\right) h_{\psi}^{-1} \partial_{\psi} \ln N_{i}^{2} T_{i}\right] \\
& \times\left(B_{\varphi}^{2} / B_{\chi}^{2}\right)\left[4 U_{\chi, i}-2.5\left(T_{i} / e_{i} B_{\varphi}\right) h_{\psi}^{-1} \partial_{\psi} \ln N_{i}^{2} T_{i}\right] \\
& \times \oint b^{(1)} \partial_{\chi} n^{(1)} d \chi .
\end{aligned}
$$

We have made use of $\partial_{\chi} t_{i}^{(1)}=-2 \partial_{\chi} n_{i}^{(1)}$ [Eq. (45), Ref. 12] and (repeatedly) of Eq. (15) to obtain this expression.

(ii) The contribution from the gyrostress tensor is 


$$
\begin{aligned}
\oint J B & \nabla \cdot \Pi_{3-4, i} \cdot \hat{n} d \chi \\
= & 0.4\left(m_{i} / e_{i}\right) h_{\varphi} B_{\varphi} \partial_{\psi}\left[\left(P_{i} / B_{\chi}\right) U_{\chi, i} \oint b^{(1)} \partial_{\chi} n^{(1)} d \chi\right] \\
& -2\left(m_{i} / e_{i}\right) h_{\varphi} B_{\varphi}\left\{\left(P_{i} / B_{\varphi}\right) \partial_{\psi} U_{\varphi, i}+B_{\chi}^{-1} \partial_{\psi} P_{i}\right. \\
& \left.\times\left[\left(T_{i} / e_{i} B_{\varphi}\right) h_{\psi}^{-1} \partial_{\psi} \ln T_{i} N_{i}^{2}-U_{\chi, i}\right]\right\} \\
& \times \oint b^{(1)} \partial_{\chi} n^{(1)} d \chi .
\end{aligned}
$$

A cancellation similar to that mentioned below Eq. (16) has taken place in the first term; thus, the Braginskii formulation again would lead to an overestimate of the novel terms in the parallel momentum equation.

(iii) The contribution from the perpendicular stress tensor is simply

$$
\oint J B \nabla \cdot \Pi_{1-2, i} \cdot \hat{n} d \chi=-2 \pi h_{\varphi} B_{\varphi} \partial_{\psi}\left(\eta_{2, i} h_{\chi} h_{\psi}^{-1} \partial_{\psi} U_{\varphi, i}\right) .
$$

(iv) The contribution from inertia can be written as

$$
\begin{aligned}
\oint & J B m_{i} N_{i}\left(\partial_{t}+\mathbf{U}_{i} \cdot \nabla\right) \mathbf{U}_{i} \cdot \hat{n} d \chi \\
= & 2 \pi m_{i} N_{i} h_{\chi}\left(B_{\varphi} / B_{\chi}\right) \partial_{t} U_{\varphi, i}-m_{i} N_{i} \\
& \times\left\{U_{\varphi, i}^{2}-2 B_{\chi}^{-2}\left(B_{\chi} U_{\varphi, i}-B_{\varphi} U_{\chi, i}\right)\right. \\
& \left.\times\left[B_{\varphi} U_{\chi, i}-\left(T_{i} / e_{i}\right) h_{\psi}^{-1} \partial_{\psi} \ln T_{i} N_{i}^{2}\right]\right\} \\
& \times \oint b^{(1)} \partial_{\chi} n^{(1)} d \chi .
\end{aligned}
$$

It is interesting to note that the contributions from inertia, gyroviscosity, and perpendicular viscosity combine into the relatively simple result:

$$
\begin{aligned}
\oint J B & \left.m_{i} N_{i}\left(\partial_{t}+\mathbf{U}_{i} \cdot \boldsymbol{\nabla}\right) \mathbf{U}_{i}+\boldsymbol{\nabla} \cdot\left(\boldsymbol{\Pi}_{3-4, i}+\mathbf{\Pi}_{1-2, i}\right)\right] \cdot \hat{n} d \chi \\
= & -2 m_{i} N_{i}\left[\frac{T_{i}}{e_{i} B_{\chi}} h_{\psi}^{-1} \frac{\partial U_{\varphi, i}}{\partial \psi}+\frac{1}{2} U_{\varphi, i}^{2}+\frac{B_{\varphi}}{B_{\chi}^{2}} h_{\psi}^{-1} \frac{\partial V}{\partial \psi}\right. \\
& \left.\times\left(U_{\chi}-\frac{T_{i}}{e_{i} B_{\varphi}} h_{\psi}^{-1} \frac{\partial \ln N_{i}^{2} T_{i}}{\partial \psi}\right)\right] \\
& \times \oint b^{(1)} \partial_{\chi} n^{(1)} d \chi+\oint B_{\varphi} h_{\chi} J_{\psi} d \chi ;
\end{aligned}
$$

the time derivative in Eq. (20) and the $\eta_{2, i}$ term in (19c) have eliminated each other in view of the ambipolarity equation (16). Finally, Eqs. (21) and (19a) can be combined into (within an error margin of less than four percent):

$$
\begin{aligned}
\oint J B & {\left[m_{i} N_{i}\left(\partial_{t}+\mathbf{U}_{i} \cdot \boldsymbol{\nabla}\right) \mathbf{U}_{i}+\nabla \cdot \Pi_{i}\right] \cdot \hat{n} d \chi } \\
= & 3 \eta_{0, i} h_{\chi}^{-1}\left[U_{\chi, i}+1.83\left(e_{i} B_{\varphi}\right)^{-1} h_{\psi}^{-1} \partial_{\psi} T_{i}\right] \oint\left(\partial_{\chi} b^{(1)}\right)^{2} d \chi-2 m_{i} N_{i}\left\{\left(T_{i} / e_{i} B_{\chi}\right) h_{\psi}^{-1} \partial_{\psi} U_{\varphi, i}+0.5 U_{\varphi, i}^{2}\right. \\
& \left.-\left(B_{\varphi} / B_{\chi}\right) U_{\varphi, i}\left[U_{\chi, i}-\left(T_{i} / e_{i} B_{\varphi}\right) h_{\psi}^{-1} \partial_{\psi} \ln N_{i}^{2} T_{i}\right]+1.90\left(B_{\varphi} / B_{\chi}\right)^{2}\left[U_{\chi, i}-0.8\left(T_{i} / e_{i} B_{\varphi}\right) h_{\psi}^{-1} \partial_{\psi} \ln N_{i}^{1.6} T_{i}\right]^{2}\right\} \\
& \times \oint b^{(1)} \partial_{\chi} n^{(1)} d \chi+B_{\varphi} h_{\chi} \oint J_{\psi} d \chi=0 .
\end{aligned}
$$

Equations (22) and (17) lead to the result (4) for large aspect ratio tokamaks with circular cross sections.

\section{DISCUSSION}

We have obtained, for the first time, the complete equations describing the poloidal and toroidal rotations of a plasma within the framework of neoclassical theory. Equations (16) and (22), supplemented by Eq. (15), are valid for large aspect ratio tokamaks whose cross sections may be noncircular (weak ellipticity, triangularity, etc.). Equations (1) and (4) follow for circular cross sections. Our results have been derived in the framework of the two-fluid equations; they apply therefore to the high collisionality regime. The Mikhailowskii-Tsypin corrections ${ }^{3}$ lower the contribu- tions of gyroviscosity in both the toroidal momentum (equivalently the ambipolarity) equation and the poloidal (equivalently the parallel) momentum equation that would otherwise result from Braginskii's tensor ${ }^{1}$ by one order of magnitude [see the comments below Eqs. (16) and (19b)]. The remaining contribution to the ambipolarity equation is obtained from the perpendicular stress tensor and coincides with the result derived earlier by Hinton and Wong ${ }^{5}$ and by Connor et al. ${ }^{6}$ Novel contributions to the weighted parallel momentum equation have also been obtained. Those are important if the parameter $\Lambda_{1}$ [Eq. (18)], introduced for the first time in Ref. 12, is $O(1 / 3)$ or larger-as results from the comparison of the last term of Eq. (4) to $1.83\left(e_{i} B_{\varphi}\right)^{-1} \partial T_{i} / \partial r$-or if the ratio $U_{\varphi, i} / c_{i}$ of the toroidal velocity to the ion thermal speed is $O\left(3 \hat{\nu}_{i}^{-1}\right)$-as results 
from the comparison of the second term on the right-hand side of Eq. (4) to the left-hand side; here $\hat{\nu}_{i}=q R \nu_{i} / c_{i}$ is the relevant collisionality parameter.

It was shown in Refs. 17 and 18 that the (sub)neoclassical heat flux can assume identical values for three distinct ion temperature length scales $L_{T, i}=\left(\partial_{r} \ln T_{i}\right)^{-1}$ if the safety factor is larger than $\sqrt{5}$. Equation (4) is a cubic with respect to $U_{\theta, i}$. We therefore also expect bifurcated solutions under certain conditions. This will be the subject of another paper.

We now briefly discuss the relevance of the above results to some experiments and suggest a further useful extension of the theory.

(1) In the absence of any external momentum source and of friction, Eq. (1) predicts a stationary toroidal rotation velocity gradient,

$$
\partial_{r} U_{\varphi, i}(r)=0.107 k q^{2}\left(\partial_{r} \ln T_{i}\right)^{2}\left(T_{i} / e_{i} B_{\theta}\right),
$$

if $\Lambda_{1} \ll 1$, since $U_{\theta, i}=k\left(T_{i} / e_{i} B_{\varphi}\right) \partial_{r} \ln T_{i}$ and $Q^{2} / S^{2} \ll 1$ in that case. In the Pfirsch-Schlüter regime, $k_{\mathrm{PS}}=-1.83$ [see Eq. (4)] and the toroidal velocity gradient is negative if $B_{\theta}$ $>0$ or positive if $B_{\theta}<0$; the toroidal velocity in the confined plasma will then be in the direction of the plasma current (codirection) if it vanishes on the last closed flux surface. In the banana regime, ${ }^{2} k_{b}=1.17$ and the toroidal velocity gradient changes sign. These predictions are consistent with experimental results from $\mathrm{PLT}^{7}{ }^{7}$ which indicate that, without beam injection, a small but consistent toroidal rotation exists in the direction of the plasma current in the plasma periphery but in the direction opposite to the current in the plasma center. For the purpose of an analytical estimate, we approximate $q \propto r^{2}, B_{\theta} \propto r^{-1}$, and $T \propto \exp (-\lambda r)$ at the plasma periphery. Introducing the PLT experimental parameters $B$ $=2.5 \mathrm{~T}, I_{p}=450 \mathrm{kA}, R_{0}=1.32 \mathrm{~m}$, and $a=0.40 \mathrm{~m}$ (limiter radius) as well as the values $T_{i} \approx 200 \mathrm{eV}$ and $\left|L_{T}\right| \approx 0.125 \mathrm{~m}$ measured at the radius $r \equiv r_{1}=0.3 \mathrm{~m}$, the above equationwhere we replace $q\left(r_{1}\right) \approx 2.13, B_{\theta}\left(r_{1}\right) \approx 0.27 \mathrm{~T}$ and $\left(r / r_{1}\right)^{5}$ $\approx \exp \left[5\left(r-r_{1}\right) / r_{1}\right]$ in the domain $r \in\left[r_{1}, a\right]$ — can readily be integrated to yield

$$
\begin{aligned}
U_{\varphi, r_{1}}-U_{\varphi, a} \approx & 0.66 \times 10^{3} \lambda^{2} r_{1}\left(5-\lambda r_{1}\right)^{-1} \\
& \times\left\{\exp \left[\left(5-\lambda r_{1}\right)\left(a / r_{1}-1\right)\right]-1\right\} \\
\approx & 0.7 \times 10^{4} \mathrm{~ms}^{-1},
\end{aligned}
$$

which compares well with the measured value $U_{\varphi, r_{1}} \approx 1$ $\times 10^{4} \mathrm{~ms}^{-1}$. We note that the collisionality parameter $\hat{\nu}_{i}$ $\sim 0.15$ if $N_{i} Z_{\text {eff }}=3 \times 10^{19} \mathrm{~m}^{-3}$ at $r=r_{1}$; the plasma is therefore mostly in the Pfirsch-Schlüter collisionality regime $\left(\hat{\nu}_{i} \geqslant 0.222\right)^{16}$ in the domain under consideration.

(2) High edge collisionalities are achieved in the radiative Improved confinement mode ${ }^{21}$ of TEXTOR 94 and in an ALCATOR C-MOD. ${ }^{22}$

Figure 3 of Ref. 21 shows that $T_{i} \sim T_{e} \approx 50 \mathrm{eV}, N_{e}$ $\approx 8.0 \times 10^{18} \mathrm{~m}^{-3}, L_{T e} \approx 3 \times 10^{-2} \mathrm{~m}$ and $L_{N e} \approx 1 \times 10^{-2} \mathrm{~m}$ at the last closed flux surface of $I_{p}=400 \mathrm{kA}, B=2.25 \mathrm{~T}, \bar{N}_{e, o}$ $\approx 6.0 \times 10^{19} \mathrm{~m}^{-3}$ discharges with auxiliary heating at the periphery of which about $70 \%$ of the total power is radiated, owing to either neon or argon seeding (note that this line average density corresponds to Greenwald's limit; ${ }^{23}$ auxil- iary heating is usually achieved with neutral beam coinjection and ion cyclotron waves). Since $R_{0}=1.75 \mathrm{~m}$ and $a$ $=0.46 \mathrm{~m}$ in TEXTOR-94, the edge collisionality parameter $\hat{\nu}_{i}$ in those deuterium discharges was

$$
\hat{\nu}_{i} \approx 2.7,
$$

if we assume $Z_{\text {eff }}=2$; the assumption of high collisionality ( $\hat{\nu}_{i}>0.222$ ) is thus verified. The parameter $\Lambda_{1}$ in turn was

$$
\Lambda_{1} \approx 0.5 \text {. }
$$

Under those conditions, deviations from the conventional neoclassical result and even bifurcated solutions can be expected for the poloidal velocity $U_{\theta, i}$ at the edge of RI mode TEXTOR discharges. A detailed study will be the subject of another paper.

Figures 8 and 9 of Ref. 22 show that $T_{i} \approx 50 \mathrm{eV}$, $N_{e} \approx 4.0 \times 10^{19} \mathrm{~m}^{-3}, L_{\mathrm{Ti}} \sim 2 \times 10^{-2} \mathrm{~m}$ and $L_{\mathrm{Ne}} \approx 3 \times 10^{-2} \mathrm{~m}$ at the radius $r=0.20 \mathrm{~m}$ of $B=5.3 \mathrm{~T}, q(r=0.20 \mathrm{~m}) \approx 4.25$, $N_{e, 0} \approx 1.3 \times 10^{20} \mathrm{~m}^{-3}$ Ohmically heated deuterium discharges. Since $R_{0}=0.67 \mathrm{~m}$ in the ALCATOR C-MOD, the edge collisionality parameter $\hat{\nu}_{i}$ was

$$
\hat{\nu}_{i} \approx 6.25,
$$

if we again assume $Z_{\text {eff }}=2$. Finally, the parameter $\Lambda_{1}$ was

$$
\Lambda_{1} \approx 0.84 \text {. }
$$

The conclusions that we have arrived at for the TEXTOR-94 RI mode discharges are therefore a fortiori valid for those ALCATOR C-MOD Ohmic discharges.

(3) As toroidal velocities are often observed to be of the order of the sound speed, the scaling relations (11) and (12) underlying the theory may have to be modified. It is expected that increasing the ratio $U_{\varphi, i} / c_{i}$ from $\mu$ to 1 via an increase of the ratio $a_{i} / L_{\perp}$ from $\mu^{2}$ to $\mu$ would bring the inertia term in competition with the other terms in the ambipolarity equation (8).

\section{ACKNOWLEDGMENTS}

We thank U. Daybelge for useful discussions.

The work of C. Yarim was partially financed in the framework of the cooperation agreement between the Scientific and Technical Research Council of the Republic of Turkey (TÜBITAK) and the Forschungszentrum Jülich GmbH. C. Yarim wishes to thank the board of directors of the Tincel Foundation for granting him a supplementary fellowship.

\section{APPENDIX A: THE STRESS TENSORS AND THE HEAT FLUXES}

In a strongly magnetized plasma $\left(\nu_{i} / \Omega_{i} \ll 1\right)$, the stress tensor can be split into three parts.

(i) The parallel stress tensor reads as

$$
\begin{aligned}
\boldsymbol{\Pi}_{0, i}= & -3 \eta_{0, i}[\hat{n} \hat{n}-(1 / 3) \mathbf{I}]\left\{\left[\hat{n} \cdot \boldsymbol{\nabla} \mathbf{U}_{i} \cdot \hat{n}-(1 / 3) \boldsymbol{\nabla} \cdot \mathbf{U}_{i}\right]\right. \\
& \left.+\left(2 / 5 P_{i}\right)\left[\hat{n} \cdot \boldsymbol{\nabla} \mathbf{q}_{i} \cdot \hat{n}-(1 / 3) \boldsymbol{\nabla} \cdot \mathbf{q}_{i}\right]\right\}-1.84 \eta_{0, i} \\
& \times[\hat{n} \hat{n}-(1 / 3) \mathbf{I}]\left(2 / 5 P_{i}\right)\left[\hat{n} \cdot \boldsymbol{\nabla}\left(\mathbf{q}_{i}-\mathbf{q}_{i}^{*}\right) \cdot \hat{n}\right. \\
& -(1 / 3) \boldsymbol{\nabla} \cdot\left(\mathbf{q}_{i}-\mathbf{q}_{i}^{*}\right)+(1 / 3) \mathbf{q}_{i} \cdot \boldsymbol{\nabla} \ln P_{i} \\
& \left.-(2 / 3) \mathbf{q}_{i} \cdot \boldsymbol{\nabla} \ln T_{i}\right],
\end{aligned}
$$


to a sufficient accuracy. Equation (A1) is a simplified form resulting from Eqs. (4.9), (4.16), and (4.17) of Ref. 3 [for our purpose, the terms $F\|q\|$ and $\left(2 q_{\|}-q_{\|}^{*}\right) \boldsymbol{\nabla}_{\|} T$ appearing in Eq. (4.17) can be neglected; moreover, the parenthesis starting before $M \mathbf{F} \cdot \mathbf{q} / T$ should close behind $\boldsymbol{\nabla} \ln T] . \eta_{0, i}=0.96 P_{i} \nu_{i}^{-1}$. The heat fluxes $\mathbf{q}_{i}$ and $\mathbf{q}_{i}^{*}$ are given below in (iv).

(ii) The gyrostress tensor is

$$
\begin{aligned}
\boldsymbol{\Pi}_{3-4, i}= & -\eta_{3, i}\left\{(\hat{p} \hat{p}-\hat{b} \hat{b})\left[\hat{b} \cdot \boldsymbol{\nabla} \mathbf{U}_{i} \cdot \hat{p}+\hat{p} \cdot \boldsymbol{\nabla} \mathbf{U}_{i} \cdot \hat{b}+\left(2 / 5 P_{i}\right)\left(\hat{b} \cdot \boldsymbol{\nabla} \mathbf{q}_{i} \cdot \hat{p}+\hat{p} \cdot \boldsymbol{\nabla} \mathbf{q}_{i} \cdot \hat{b}\right)\right]\right. \\
& -(\hat{p} \hat{b}+\hat{b} \hat{p})\left[\hat{p} \cdot \boldsymbol{\nabla} \mathbf{U}_{i} \cdot \hat{p}-\hat{b} \cdot \boldsymbol{\nabla} \mathbf{U}_{i} \cdot \hat{b}+\left(2 / 5 P_{i}\right)\left(\hat{p} \cdot \boldsymbol{\nabla} \mathbf{q}_{i} \cdot \hat{p}-\hat{b} \cdot \boldsymbol{\nabla} \mathbf{q}_{i} \cdot \hat{b}\right)\right]+2(\hat{p} \hat{n}+\hat{n} \hat{p})\left[\hat{b} \cdot \boldsymbol{\nabla} \mathbf{U}_{i} \cdot \hat{n}+\hat{n} \cdot \boldsymbol{\nabla} \mathbf{U}_{i} \cdot \hat{b}\right. \\
& \left.\left.+\left(2 / 5 P_{i}\right)\left(\hat{b} \cdot \boldsymbol{\nabla} \mathbf{q}_{i} \cdot \hat{n}+\hat{n} \cdot \boldsymbol{\nabla} \mathbf{q}_{i} \cdot \hat{b}\right)\right]-2(\hat{b} \hat{n}+\hat{n} \hat{b})\left[\hat{p} \cdot \boldsymbol{\nabla} \mathbf{U}_{i} \cdot \hat{n}+\hat{n} \cdot \boldsymbol{\nabla} \mathbf{U}_{i} \cdot \hat{p}+\left(2 / 5 P_{i}\right)\left(\hat{p} \cdot \boldsymbol{\nabla} \mathbf{q}_{i} \cdot \hat{n}+\hat{n} \cdot \boldsymbol{\nabla} \mathbf{q}_{i} \cdot \hat{p}\right)\right]\right\},
\end{aligned}
$$

to a sufficient accuracy. $\eta_{3, i}=P_{i} / 2 \Omega_{i}$. The index 3-4 refers to Braginskii's coefficients $\eta_{3}$ and $\eta_{4}=2 \eta_{3}$. (It is useful to write $\boldsymbol{\Pi}_{3-4, i}, \boldsymbol{\Pi}_{0, i}$, and $\boldsymbol{\Pi}_{1-2, i}$ in full in order to compare easily the relative orders of magnitude of the different contributions.)

(iii) The perpendicular stress tensor is

$$
\begin{aligned}
\boldsymbol{\Pi}_{1-2, i}= & -\eta_{1, i}\left\{(\hat{p} \hat{p}-\hat{b} \hat{b})\left[\hat{p} \cdot \boldsymbol{\nabla} \mathbf{U}_{i} \cdot \hat{p}-\hat{b} \cdot \boldsymbol{\nabla} \mathbf{U}_{i} \cdot \hat{b}+\left(2 / 5 P_{i}\right)\left(\hat{p} \cdot \boldsymbol{\nabla} \mathbf{q}_{i} \cdot \hat{p}-\hat{b} \cdot \boldsymbol{\nabla} \mathbf{q}_{i} \cdot \hat{b}\right)\right]+(\hat{p} \hat{b}+\hat{b} \hat{p})\right. \\
& \times\left[\hat{p} \cdot \boldsymbol{\nabla} \mathbf{U}_{i} \cdot \hat{b}+\hat{b} \cdot \boldsymbol{\nabla} \mathbf{U}_{i} \cdot \hat{p}+\left(2 / 5 P_{i}\right)\left(\hat{p} \cdot \boldsymbol{\nabla} \mathbf{q}_{i} \cdot \hat{b}+\hat{b} \cdot \boldsymbol{\nabla} \mathbf{q}_{i} \cdot \hat{p}\right)\right]+4(\hat{p} \hat{n}+\hat{n} \hat{p})\left[\hat{p} \cdot \boldsymbol{\nabla} \mathbf{U}_{i} \cdot \hat{n}+\hat{n} \cdot \boldsymbol{\nabla} \mathbf{U}_{i} \cdot \hat{p}\right. \\
& \left.\left.+\left(2 / 5 P_{i}\right)\left(\hat{p} \cdot \boldsymbol{\nabla} \mathbf{q}_{i} \cdot \hat{n}+\hat{n} \cdot \boldsymbol{\nabla} \mathbf{q}_{i} \cdot \hat{p}\right)\right]+4(\hat{b} \hat{n}+\hat{n} \hat{b})\left[\hat{b} \cdot \boldsymbol{\nabla} \mathbf{U}_{i} \cdot \hat{n}+\hat{n} \cdot \boldsymbol{\nabla} \mathbf{U}_{i} \cdot \hat{b}+\left(2 / 5 P_{i}\right)\left(\hat{b} \cdot \boldsymbol{\nabla} \mathbf{q}_{i} \cdot \hat{n}+\hat{n} \cdot \boldsymbol{\nabla} \mathbf{q}_{i} \cdot \hat{b}\right)\right]\right\},
\end{aligned}
$$

to a sufficient accuracy. $\eta_{1, i}=3 P_{i} \nu_{i} / 10 \Omega_{i}^{2}$. The index 1-2 refers to Braginskii's coefficients $\eta_{1}, \eta_{2}=4 \eta_{1}$.

(iv) The heat flux $\mathbf{q}_{i}$ is given by ${ }^{1,3}$

$$
\begin{aligned}
\mathbf{q}_{i}= & -\left(P_{i} / m_{i}\right)\left[3.9 \nu_{i}^{-1} \hat{n} \hat{n} \cdot \boldsymbol{\nabla} T_{i}-(5 / 2) \Omega_{i}^{-1} \hat{n} \times \nabla T_{i}\right. \\
& \left.-2 \nu_{i} \Omega_{i}^{-2} \hat{n} \times\left(\hat{n} \times \nabla T_{i}\right)\right] \\
= & -\left(P_{i} / m_{i}\right)\left\{3.9 \nu_{i}^{-1} \hat{n}\left(B_{\chi} h_{\chi}^{-1} / B\right) \partial_{\chi} T_{i}\right. \\
& -\Omega_{i}^{-1} \hat{b}\left[(5 / 2) h_{\psi}^{-1} \partial_{\psi} T_{i}-2\left(\nu_{i} / \Omega_{i}\right)\left(B_{\varphi} h_{\chi}^{-1} / B\right) \partial_{\chi} T_{i}\right] \\
& \left.+\Omega_{i}^{-1} \hat{p}\left[(5 / 2)\left(B_{\varphi} h_{\chi}^{-1} / B\right) \partial_{\chi} T_{i}+2\left(\nu_{i} / \Omega_{i}\right) h_{\psi}^{-1} \partial_{\psi} T_{i}\right]\right\},
\end{aligned}
$$

to a sufficient accuracy. Furthermore,

$$
\mathbf{q}_{i}^{*}=1.04\left(P_{i} / m_{i}\right) \nu_{i}^{-1} \hat{n} \hat{n} \cdot \nabla T_{i}
$$

\section{APPENDIX B: OUTLINE OF THE DERIVATION OF $\Pi_{i}$}

The tensors defined by Eqs. (A1)-(A3) differ from those given by Braginskii ${ }^{1}$ and $\mathrm{Balescu}^{24}$ by the terms involving the heat flux $\mathbf{q}_{i}$ and $\mathbf{q}_{i}^{*}$. Those terms are necessary for the two-fluid equations to reproduce the expression $U_{\theta, i}$ $=-1.83 \partial_{r} T_{i} / e_{i} B_{\varphi}$ predicted by a kinetic analysis ${ }^{2}$ (Hazeltine's coefficient $k=-2.1$ for the high collisionality regime has later been modified slightly). They also play an important role both in the ambipolarity (identically the toroidal momentum) equation-where the Braginskii formulation leads to overestimating the effect of gyroviscosity by one order of magnitude - and in the generalized parallel momentum equation (which provides the poloidal velocity). It is therefore important to show how they arise.

Following Grad's 21-moment approximation, ${ }^{25}$ the ion distribution function in a pure plasma is

$$
\begin{aligned}
F_{i}= & N_{i}\left(\pi v_{\mathrm{th}, i}^{2}\right)^{-3 / 2} \exp \left(-x^{2}\right)\left[1+\frac{4}{5}\left(x^{2}-\frac{5}{2}\right) \frac{\mathbf{q}_{i}}{v_{\mathrm{th}, i} P_{i}} \cdot \mathbf{x}\right. \\
& +\frac{8}{35}\left(x^{4}-7 x^{2}+\frac{35}{4}\right) \frac{\mathbf{r}_{i}}{v_{\mathrm{th}, i}^{3} P_{i}} \cdot \mathbf{x}+\frac{\Pi_{i}}{P_{i}}:\left(\mathbf{x x}-\frac{\mathrm{I}}{3} x^{2}\right) \\
& \left.+\frac{4}{7}\left(x^{2}-\frac{7}{2}\right) \frac{\boldsymbol{\sigma}_{i}}{v_{\mathrm{th}, i}^{2} P_{i}}:\left(\mathbf{x x}-\frac{1}{3} x^{2}\right)\right],
\end{aligned}
$$

in the limit $m_{e} / m_{i} \rightarrow 0$. Here, $v_{\mathrm{th}, i}=\left(2 T_{i} / m_{i}\right)^{1 / 2}$ (we note that $v_{\mathrm{th}, i}=\sqrt{2} c_{i}$, where $c_{i}$ is the thermal velocity defined in the main text) and $\mathbf{x}=\left(v-\mathbf{U}_{i}\right) / v_{\text {th }, i}, \mathbf{v}$ being the velocity field of the particles:

$$
N_{i} \mathbf{U}_{i}=\int \mathbf{v} F_{i} d^{3} \mathbf{v}
$$

is the ion mean velocity,

$$
\mathbf{q}_{i}=\left(m_{i} / 2\right) \int\left|\mathbf{v}-\mathbf{U}_{i}\right|^{2}\left(\mathbf{v}-\mathbf{U}_{i}\right) F_{i} d^{3} \mathbf{v}
$$

is the heat flux,

$$
\boldsymbol{\Pi}_{i}=m_{i} \int\left[\left(\mathbf{v}-\mathbf{U}_{i}\right)\left(\mathbf{v}-\mathbf{U}_{i}\right)-\frac{1}{3}\left|\mathbf{v}-\mathbf{U}_{i}\right|^{2}\right] F_{i} d^{3} \mathbf{v}
$$

is the stress tensor;

$\mathbf{r}=\left(m_{i} / 4\right) \int\left[\left|\mathbf{v}-\mathbf{U}_{i}\right|^{2}-7 v_{\mathrm{th}, i}^{2}\right]\left|\mathbf{v}-\mathbf{U}_{i}\right|^{2}\left(\mathbf{v}-\mathbf{U}_{i}\right) F_{i} d^{3} \mathbf{v}$

and

$$
\begin{aligned}
\boldsymbol{\sigma}_{i}= & \left(m_{i} / 2\right) \int\left[\left(\mathbf{v}-\mathbf{U}_{i}\right)\left(\mathbf{v}-\mathbf{U}_{i}\right)-\frac{\mathrm{I}}{3}\left|\mathbf{v}-\mathbf{U}_{i}\right|^{2}\right] \\
& \times\left[\left|\mathbf{v}-\mathbf{U}_{i}\right|^{2}-\frac{7}{2} v_{\mathrm{th}, i}^{2}\right] F_{i} d^{3} \mathbf{v}
\end{aligned}
$$


are higher odd and even moments. We note that the tensors (B4) and (B6) are symmetric and that their trace is zero, reducing the number of tensorial components to ten in total.

Multiplying the Vlasov-Boltzmann equation,

$$
\frac{\partial F_{i}}{\partial t}+\mathbf{v} \cdot \nabla F_{i}+\frac{e_{i}}{m_{i}}(\mathbf{E}+\mathbf{v} \times \mathbf{B}) \cdot \frac{\partial F_{i}}{\partial \mathbf{v}}=C\left(F_{i}, F_{i}\right)
$$

(electron-ion collisions are neglected in the limit $\left.m_{e} / m_{i} \rightarrow 0\right)$ by $\Phi_{1}=m_{i}\left[\left(\mathbf{v}-\mathbf{U}_{i}\right)\left(\mathbf{v}-\mathbf{U}_{i}\right)-\mathbf{I} v_{\text {th }, i}^{2} / 3\right]$, respectively, $\Phi_{2}=\left(m_{i} / 2\right)\left[\left(\mathbf{v}-\mathbf{U}_{i}\right)\left(\mathbf{v}-\mathbf{U}_{i}\right)-\mathbf{I} v_{\mathrm{th}, i}^{2} / 3\right]\left[\left|\mathbf{v}-\mathbf{U}_{i}\right|^{2}\right.$ $\left.-(7 / 2) v_{\mathrm{th}, i}^{2}\right]$, and integrating over velocity space leads to

$$
\left\{\frac{2}{5} \boldsymbol{\nabla} \mathbf{q}_{i}+P_{i} \boldsymbol{\nabla} \mathbf{U}_{i}-\Omega_{i}(\hat{p} \hat{b}-\hat{b} \hat{p}) \cdot \Pi_{i}\right\}=\int \Phi_{1} C\left(F_{i}, F_{i}\right) d^{3} \mathbf{v},
$$

respectively,

$$
\left\{\boldsymbol{\nabla}\left(\frac{7}{10} v_{\mathrm{th}, i}^{2} \mathbf{q}_{i}+\frac{2}{5} \mathbf{r}_{i}\right)-\Omega_{i}(\hat{p} \hat{b}-\hat{b} \hat{p}) \cdot \boldsymbol{\sigma}\right\}=\int \Phi_{2} C\left(F_{i}, F_{i}\right) d^{3} \mathbf{v} .
$$

Here the curly bracket indicates the following operation on the tensors:

$$
\{A\}=A+A^{T}-(2 / 3) I \operatorname{tr} A,
$$

where $T$ is the transposed and tr the trace. The above two equations are coupled by the collision integrals that depend on both $\boldsymbol{\Pi}_{i}$ and $\boldsymbol{\sigma}_{i}$. Similarly, $\mathbf{q}_{i}$ and $\mathbf{r}_{i}$ are collisionally coupled.

The three types of terms occuring in Eqs. (B7) and (B8) scale as the transit frequency, the gyrofrequency, and the collision frequency, respectively. Hence, in the high collisionality regime, the equations

$$
\begin{aligned}
& \left\{(\hat{p} \hat{b}-\hat{b} \hat{p}) \cdot \boldsymbol{\Pi}_{i}\right\}^{(0)}=0, \\
& \left\{\frac{2}{5 P_{i}} \boldsymbol{\nabla} \mathbf{q}_{i}+\boldsymbol{\nabla} \mathbf{U}_{i}\right\}^{(0)}=\frac{\Omega_{i}}{P_{i}}\left\{(\hat{p} \hat{b}-\hat{b} \hat{p}) \cdot \Pi_{i}\right\}^{(1)},
\end{aligned}
$$

and

$$
\begin{aligned}
& \left\{\frac{2}{5 P_{i}} \boldsymbol{\nabla} \mathbf{q}_{i}+\boldsymbol{\nabla} \mathbf{U}_{i}\right\}^{(1)} \\
& \quad=\frac{\Omega_{i}}{P_{i}}\left\{(\hat{p} \hat{b}-\hat{b} \hat{p}) \cdot \Pi_{i}\right\}^{(2)}+\frac{1}{P_{i}} \int \Phi_{i} C\left(F_{i}, F_{i}\right) d^{3} \mathbf{v},
\end{aligned}
$$

which, together with similar equations for $\boldsymbol{\sigma}_{i}$, allow us to obtain the structure of the tensors $\boldsymbol{\Pi}_{0, i}\left(\propto \nu_{i}^{-1}\right), \boldsymbol{\Pi}_{3-4, i}$ $\left(\propto \Omega_{i}^{-1}\right)$, and $\Pi_{1-2, i}\left(\propto \nu_{i} \Omega_{i}^{-2}\right)$. The combination of the heat flux and velocity gradients in (B9) and (B10) explains the structure of the Mikhailovskii-Tsypin stress tensors. Noting that the heat flux is of order $\left(a_{i} / L_{\perp}\right) c_{i} P_{i}$ [cf. Eq. (A4), where $\partial^{2} T_{i} / \partial \chi^{2}$ and hence $\partial T_{i} / \partial \chi$ is of order $\epsilon\left(\nu_{i} / \Omega_{i}\right)$ $\times\left(q^{2} R^{2} / r L_{\perp}\right) T_{i}$ following Eq. (15)], the term $\left(2 / 5 P_{i}\right) \boldsymbol{\nabla} \mathbf{q}_{i}$ may be neglected if the fluid velocity $\mathbf{U}_{i}$ scales as the thermal velocity - as is usually assumed in homogeneous fluid theory-not if $\mathbf{U}_{i}$ scales as the diamagnetic velocity, as is the case in magnetically confined toroidal plasmas. We note finally that the origin of the parallel flux $\mathbf{q}^{*}$ in Eq. (A1) is the vector $(2 / 5) \mathbf{r}_{i}$ in Eq. (B8).

${ }^{1}$ S. I. Braginskii, in Reviews of Plasma Physics, edited by M. A. Leontovich (Consultants Bureau, New York, 1965), Vol. 1, p. 214.

${ }^{2}$ R. D. Hazeltine, Phys. Fluids 17, 961 (1974).

${ }^{3}$ A. B. Mikhailowskii and V. S. Tsypin, Beitr. Plasmaphys. 24, 335 (1984).

${ }^{4}$ J. T. Hogan, Phys. Fluids 27, 2308 (1984).

${ }^{5}$ F. L. Hinton and K. Wong, Phys. Fluids 28, 3082 (1985).

${ }^{6}$ J. W. Connor, S. C. Cowley, R. J. Hastie, and L. R. Pan, Plasma Phys. Controlled Fusion 29, 919 (1987).

${ }^{7}$ S. Suckewer, H. P. Eubank, R. J. Goldston, J. McEnerney, N. R. Sauthoff, and H. H. Towner, Nucl. Fusion 21, 1301 (1981).

${ }^{8}$ K. Brau, M. Bitter, R. J. Goldston, D. Manos, K. McGuire, and S. Suckewer, Nucl. Fusion 23, 1643 (1983).

${ }^{9}$ K. H. Burrell, R. J. Groebner, H. St. John, and R. P. Seraydarian, Nucl. Fusion 28, 3 (1988).

${ }^{10}$ W. M. Stacey and D. J. Sigmar, Phys. Fluids 28, 2800 (1985).

${ }^{11}$ W. M. Stacey, Phys. Fluids B 5, 1413 (1993).

${ }^{12}$ A. Rogister, Phys. Plasmas 1, 619 (1994).

${ }^{13}$ H. A. Claassen and H. Gerhauser, Czech. J. Phys. 49, 69 (1999).

${ }^{14}$ J. Cornelis, R. Sporken, G. van Oost, and R. R. Weynants, Nucl. Fusion 34, 171 (1994).

${ }^{15}$ J. L. Lachambre, B. Quirion, C. Boucher, R. Decoste, D. Michaud, G. Le Clair, P. Couture, A. Boileau, J. L. Gauvreau, D. Bourgoin, and J. Gunn, Nucl. Fusion 34, 1431 (1994).

${ }^{16} \mathrm{R}$. Balescu, in Transport Processes in Plasmas, Vol. 2, Neoclassical Transport (North-Holland, Amsterdam, 1988), p. 659.

${ }^{17}$ A. Rogister, Phys. Rev. Lett. 81, 3663 (1998).

${ }^{18}$ A. Rogister, Phys. Plasmas 6, 200 (1999).

${ }^{19}$ A. Rogister and D. Li, Phys. Fluids B 4, 804 (1992).

${ }^{20}$ P. H. Rutherford, Phys. Fluids 13, 482 (1970).

${ }^{21}$ B. Unterberg, A. M. Messiaen, J. Ongena, M. Brix, G. Bertschinger, J. Boedo, G. Bonheure, M. Ciotti, Th. Denner, F. Durodié, P. Dumortier, K. H. Finken, G. Fuchs, R. Jaspers, Y. M. Kim, R. Koch, L. Könen, H. R. Koslowski, A. Krämer-Flecken, A. Lyssoivan, G. Mank, G. Van Oost, A. Pospieszczyk, V. Philipps, J. Rapp, U. Samm, B. Schweer, G. Telesca, M. Z. Tokar, R. Uhlemann, P. E. Vandenplas, M. Vervier, G. Waidmann, G. Van Wassenhove, F. Weschenfelder, R. R. Weynants, and G. H. Wolf, Plasma Phys. Controlled Fusion 39, B189 (1997).

${ }^{22}$ J. E. Rice, J. L. Terry, E. S. Marmar, and F. Bombarda, Nucl. Fusion 37, 241 (1997).

${ }^{23}$ M. Greenwald, G. L. Terry, S. M. Wolfe, S. Ejima, M. G. Bell, S. M. Kaye, and G. H. Neilson, Nucl. Fusion 28, 2199 (1988).

${ }^{24} \mathrm{R}$. Balescu, in Transport Processes in Plasmas, Vol. 1, Classical Transport (North-Holland, Amsterdam, 1988), p. 255.

${ }^{25}$ H. Grad, Commun. Pure Appl. Math. 2, 311 (1949); and in Handbuch der Physik, Principles of the Kinetic Theory of Gases (Springer-Verlag, Berlin, 1958), Vol. 12, p. 205. 